\title{
Diffusion of hydrocarbons in confined media: Translational and rotational motion
}

\author{
S Y BHIDE, A V ANIL KUMAR and S YASHONATH* \\ Solid State and Structural Chemistry Unit, Indian Institute of Science, \\ Bangalore 560 012, India \\ e-mail: yashonath@sscu.iisc.ernet.in
}

\begin{abstract}
Diffusion of monatomic guest species within confined media has been understood to a good degree due to investigations carried out during the past decade and a half. Most guest species that are of industrial relevance are actually polyatomics such as, for example, hydrocarbons in zeolites. We attempt to investigate the influence of non-spherical nature of guest species on diffusion. Recent molecular dynamics (MD) simulations of motion of methane in $\mathrm{NaCaA}$ and $\mathrm{NaY}$, benzene in $\mathrm{NaY}$ and one-dimensional channels $\mathrm{AlPO}_{4}-5$, VPI-5 and carbon nanotube indicate interesting insights into the influence of the host on rotational degrees of freedom and orientational properties. It is shown that benzene in one-dimensional channels where the levitation parameter is near unity exhibits translational motion opposite to what is expected on the basis of molecular anisotropy. Rotational motion of benzene also possesses rotational diffusivities around $C_{6}$ and $C_{2}$ axes opposite to what is expected on the basis of molecular geometry. Methane shows orientational preference for $2+2$ or $1+3$ depending on the magnitude of the levitation parameter.
\end{abstract}

Keywords. Diffusion hydrocarbons; orientational preference; translationalorientational coupling; molecular dynamics.

\section{Introduction}

Understanding the diffusion of hydrocarbons within zeolites and other porous solids is of considerable importance because of their significance in the petrochemical industry. Both aliphatic and aromatic hydrocarbons are processed using zeolites. Separation of mixtures as well as cracking of hydrocarbons is achieved through the use of porous solids such as zeolites. The saving that can be achieved through the improvement in the efficiency of these catalytic and separation processes can be significant.

At a more fundamental level, the laws governing the diffusion and other properties of confined systems are barely understood unlike in the case bulk fluids. Such an understanding might lead to improvement in existing processes as well as in the design of new processes. In the past few years, detailed studies of monatomics diffusing within zeolites have been investigated in detail. Excellent reviews exist in the literature ${ }^{1-3}$. These have increased significantly our understanding of the various factors that control diffusion within zeolites of monatomics. Polyatomics such as hydrocarbons have not been investigated in detail.

The present study attempts to provide a summary of our understanding of the diffusion of polyatomics within zeolites and other porous solids in relation to the

\footnotetext{
*For correspondence
} 
orientational and rotational degrees of freedom as found from computer simulations studies. The techniques employed include kinetic Monte Carlo and molecular dynamics methods.

\section{Structure of hosts}

The equilibrium and dynamical properties of polyatomic species are studied in microporous hosts that are made up of three-dimensional network of interconnected voids and hosts that consists of voids forming one-dimensional channels. The three-dimensional (3D) host structures used are aluminosilicates like zeolites A and NaY. One-dimensional (1D) channels are aluminophosphates like $\mathrm{AlPO}_{4}-5$ and VPI-5 and carbon nanotubes.

Zeolite A crystallises in space group $F m \overline{3} c$ with a unit cell composition of $\mathrm{Na}_{32} \mathrm{Ca}_{32} \mathrm{Si}_{96} \mathrm{Al}_{96} \mathrm{O}_{384}{ }^{4}$. Sodium and calcium are extra framework cations which maintain charge neutrality in a unit cell. The void network consists of large cages, known as Otcages of approximately $11.5 \AA$ diameter interconnected via 8-membered rings of about $4.5 \AA$ diameter (see figure 1a). The unit cell length is $24.555 \AA$.

Zeolite $\mathrm{Y}$ crystallises in space group $F d \overline{3} m$ with unit cell length of $24 \cdot 85 \AA^{5}$. The crystallographic unit cell consists of $8 \alpha$ cages. Each $\alpha$ cage (diameter $11.8 \AA$ ) is connected to four neighbouring cages through a 12-membered oxygen ring (diameter $7 \cdot 5 \AA$ ) (see figure $1 \mathrm{~b}$ ). The Si/Al ratio varies in the range 1.5-3 for NaY corresponding to 6 or fewer exchangeable cations per cage.

$\mathrm{AlPO}_{4}-5$ crystallises in hexagonal space group P6/mcc with $a=13.77$ and $c=8.38 \AA^{6}$. There are 72 atoms in one unit cell. The channel does not have uniform diameter throughout (figure $1 \mathrm{c}$ and $\mathrm{d}$ ), it is narrow near $z \approx 0.25 c$ and $0.75 c$ (dia $\approx 10.02 \AA$ ) but broader in the rest of the region $(\mathrm{dia} \approx 11.37 \AA$ ).

VPI-5 crystallises in a hexagonal space group $P 6_{3} c m$ with $a=19 \cdot 0$ and $c=8 \cdot 11 \AA^{7}$. There are 108 atoms in one unit cell. The channels are elliptical. The minor and major axis lengths are not uniform throughout (figures $1 \mathrm{e}$ and $\mathrm{f}$ ). The elliptical cross-sections in the narrow $(z \approx 0.25 c)$ and the wide $(z \approx 0.75 c)$ portions of the channel have dimensions 14.44 (minor) $\times 16.31$ (major) $\AA$ and $15.92 \times 16.31 \AA$ respectively. Note that the diagonally opposite oxygens near the minor axis in the narrow part of the channel are at a distance of $14 \cdot 68 \AA$.

The carbon nanotube structure is constructed by the method of arm-chair mode of rolling the graphite sheet (see figure $1 \mathrm{~g}$ ) ${ }^{8,9}$. It is possible to have carbon nanotubes with different diameters. Here we have chosen a carbon nanotube of diameter of $10 \cdot 8 \AA$. The length of a single carbon nanotubes is taken to be $c=49.29 \AA$, which contains 640 carbon atoms. Unlike $\mathrm{AlPO}_{4}-5$ and VPI-5, the carbon nanotube has uniform diameter and a smooth and homogeneous wall throughout.

\section{Results and discussion}

\subsection{Benzene in $Y$ zeolite}

Benzene in $\mathrm{NaY}$ has been investigated using molecular dynamics (MD) and kinetic Monte Carlo ${ }^{10,11}$. The MD simulations suggested that benzene has two adsorption sites, the cation site and the window site. In the former, benzene adsorbs just above the sodium cation. It appears that there is a strong electrostatic interaction between the cation and the 
(a)
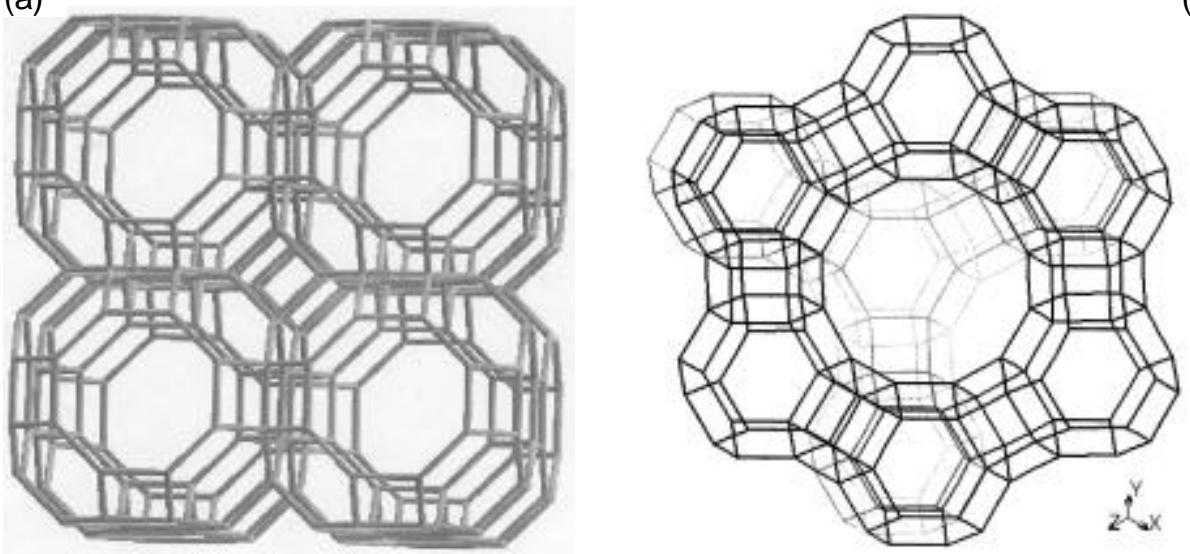

(b)

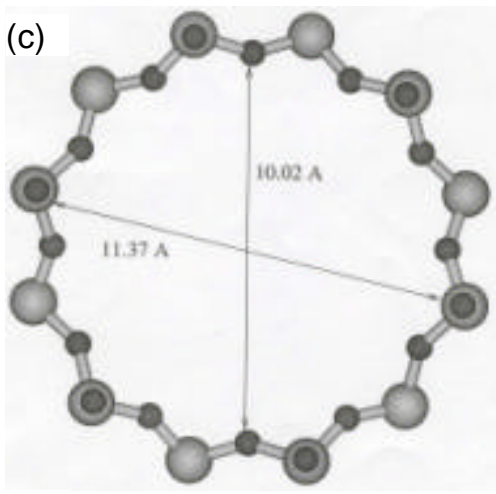

(e)
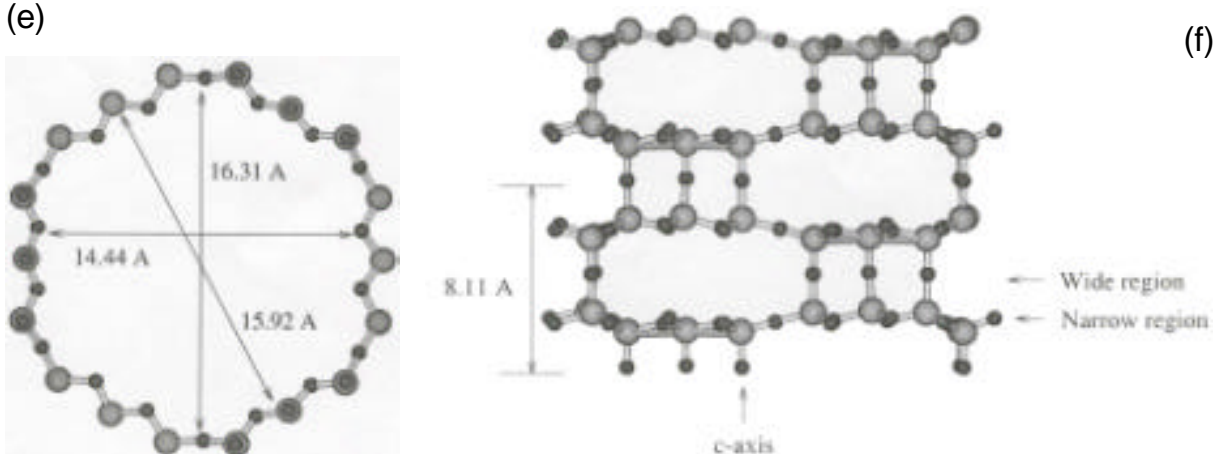

(f)

Figure 1. Schematic view of different hosts and their channel diameters. For $\mathrm{AlPO}_{4}-5$ and VPI-5, dark spheres correspond to oxygens. (a) A schematic diagram of unit cell of zeolite A showing arrangement and connectivity of $\alpha$-cages. (b) A schematic diagram of an $\alpha$-cage of zeolite Y.(c) A cross-sectional view of the $\mathrm{AlPO}_{4}-5$ channel. There are two diameters, the narrow $(10.02 \AA)$ and the broad $(11.37 \AA$ ) which correspond respectively to the 12 -ring window plane and region connecting two such window planes. (d) Vertical view of the $\mathrm{AlPO}_{4}-5$ channel with the different regions indicated along the channel. (e) Cross-sectional view of the VPI-5 channel. $14 \cdot 44 \AA$ and $16 \cdot 31 \AA$ are the minor and the major axes respectively. $15.92 \AA$ and $16 \cdot 31 \AA$ are the minor and major axes at the wide region. Wider region is bounded by the oxygens connecting two 18 -ring windows forming the channel. (f) Vertical view of the VPI-5 channel with the different regions indicated along the channel. 
(g)

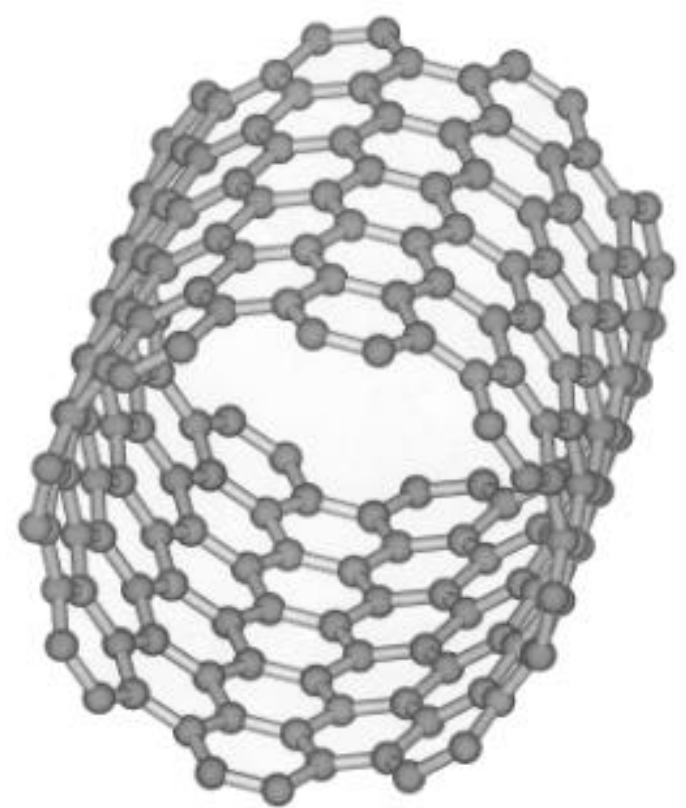

Figure 1. (g) Carbon nanotube.

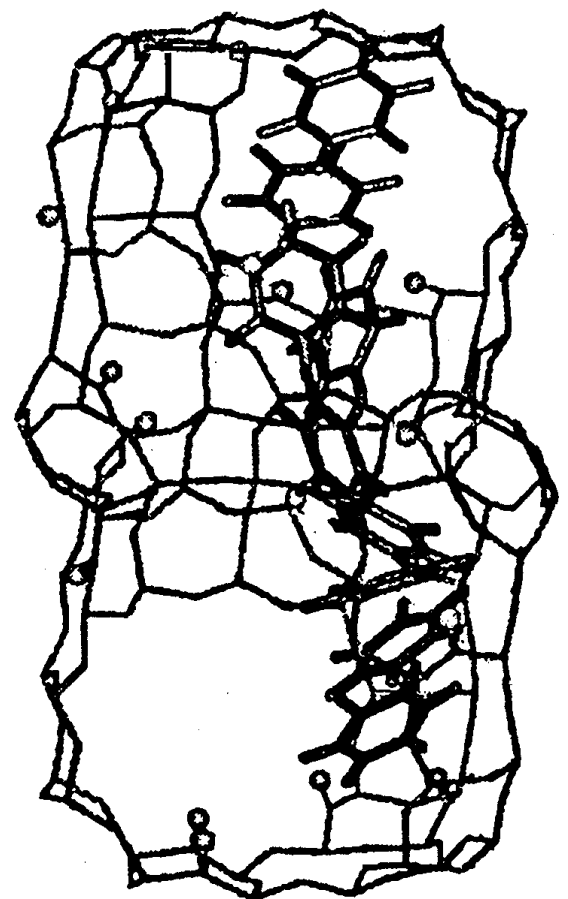

Figure 2. Minimum energy path (MEP) of benzene between two cages of HY, as calculated by constrained minimization procedure. Shaded circles represent zeolite protons (adapted from ref. 12) 
$\pi$ electron cloud. At the window site, each of the hydrogens of the benzene interact with the oxygens of the 12-ring window which interconnects two $\alpha$-cages. Again this interaction is largely electrostatic. Auerbach and coworkers ${ }^{12}$ in a detailed study investigated the nature of diffusion between cation to cation site via the window site. They obtained the minimum energy path by constrained minimization procedure. They found that benzene migrates from one adsorption site to another by means of creeping or gliding motion of benzene (see figure 2). The other motion termed the cartwheel motion had a higher activation energy $(30 \mathrm{~kJ} / \mathrm{mol})$ in comparison to gliding motion $(20 \mathrm{~kJ} / \mathrm{mol})$.

\subsection{Benzene in one-dimensional channels}

Diffusion of benzene, the simplest member of the aromatic family of hydrocarbons in one-dimensional channels $\mathrm{AlPO}_{4}-5$ and carbon nanotubes was investigated recently by classical molecular dynamics simulations ${ }^{13}$. Properties related to orientation and rotational motion were computed.

3.2a Translational motion: Variation of mean square displacement with time for benzene inside all three hosts is shown in figure 3. The mean square displacements for benzene in $\mathrm{AlPO}_{4}-5$ and VPI-5 vary linearly with time. The self-diffusivity is calculated from the slope of the straight line fit by Einstein's relation,

$$
D^{t}=\left\langle r^{2}\right\rangle / 6 t .
$$

The straight line is fitted over the range 50 to $250 \mathrm{ps}$. The calculated values of selfdiffusion coefficients are listed in table 1.

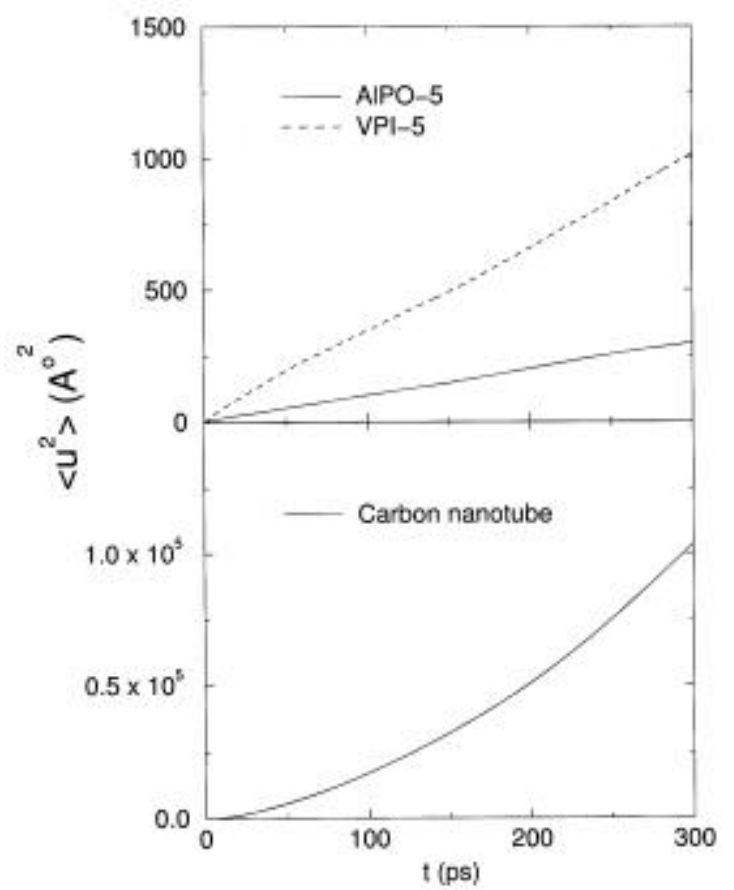

Figure 3. Mean square displacement of benzene in channels of $\mathrm{AlPO}_{4}-5, \mathrm{VPI}-5$ and carbon nanotube. 
Table 1. Self-diffusion coefficient of benzene in $1 \mathrm{D}$ channels.

\begin{tabular}{lcc}
\hline & $D^{t}\left(10^{-9} \mathrm{~m}^{2} / \mathrm{s}\right)$ & Density, $\rho(\mathrm{g} / \mathrm{cc})$ \\
\hline $\mathrm{AlPO}_{4}-5$ & 1.696 & 0.0492 \\
VPI-5 & $5 \cdot 264$ & 0.0048 \\
Liquid benzene (experimental) $^{18}$ & $2 \cdot 8$ & 0.864 \\
Liquid benzene (simulation) $^{14}$ & $3 \cdot 1$ & 0.864 \\
\hline
\end{tabular}

Table 1 also lists self-diffusivity for liquid benzene determined from experiment and by MD simulations of Linse et $a l^{14}$. It can be seen that self-diffusivity for benzene in $\mathrm{AlPO}_{4}-5$ is lower than that for benzene in bulk liquid despite the lower density in $\mathrm{AlPO}_{4}-5$. This is probably due to the hindrance to movement in $\mathrm{AlPO}_{4}-5$ due to presence of constrictions. $D^{t}$ value is the highest in VPI-5 where the density of benzene is the lowest. In carbon nanotube the $\left\langle u^{2}\right\rangle \mathrm{vs} t$ is found to be parabolic and never goes over to diffusive regime.

The VACF for benzene along the space fixed directions inside the three-channel systems suggests that diffusion along the channel axis is more facile as expected. The VACF in body-fixed frame would provide us with information about the way the motion occurs i.e. parallel to the $C_{6}$ axis or perpendicular to the $C_{6}$ axis. In figure 4 the VACF in body-fixed frame for benzene in the three hosts is plotted. The VACF exhibits some changes from that of the bulk benzene. Along the $C_{2}$ axis, we see that the motion is more hindered when benzene is confined. It is seen that in liquid benzene ${ }^{14}$, at higher temperatures, the difference between the VACF along $C_{6}$ and $C_{2}$ reduces with little or no 'cage effect'. At lower temperatures, both components of the VACF exhibit negative regions in liquid benzene suggesting 'cage effect'. In confined benzene, the $C_{6}$ component exhibits multiple minima. In liquid benzene, two minima are seen for the $C_{6}$ component. The predominance of motion parallel to $C_{6}$ or $C_{2}$ can be gleaned from the ratio $\beta=D_{\perp}^{t} / D_{\|}^{t}$. The 'self-diffusion' coefficients calculated from the unnormalized $\mathrm{VACF}$ in body-fixed frame along with $\beta$ are listed in table 2. The integration is performed up to $25 \mathrm{ps}$ for $\mathrm{ALPO}_{4}-5$ and VPI-5 and for the $50 \mathrm{ps}$ for the carbon nanotube. In bulk benzene, motion is predominantly along the $C_{2}$ axis which also has higher correlation time as compared to $C_{6}$ axis ${ }^{14}$. This would correspond to a $\beta$ greater than 1 and can be understood in terms of ratio of diameters of benzene along $C_{6}$ and $C_{2}$ directions which is around 1.6. Similar behaviour is observed in VPI-5 also where the ratio $\beta$ is around 1.83. In narrow channel such as carbon nanotube what is seen is just the reverse: $\beta$ is 0.35 suggesting that the motion occurs predominantly with the plane of benzene ring perpendicular to the direction of motion. In the case of $\mathrm{AlPO}_{4}-5$ the presence of surface roughness coupled with the modulations in the channel diameter, prevents diffusion where the benzene ring is perpendicular to the direction of translational motion in spite of the fact that the channel diameter is comparable to the carbon nanotube.

3.2b Rotational motion: The angular velocity autocorrelation function in the body fixed frame is shown in figure 5. First we note that, in all the three hosts, rotation of benzene around $C_{2}$ axis is hindered as compared to that around $C_{6}$ axis. Second, rotation around $C_{6}$ axis is easier in VPI-5 and carbon nanotube as compared to $\mathrm{AlPO}_{4}-5$. In carbon nanotube the correlation for rotation around $C_{6}$ axis persist over a significantly longer 

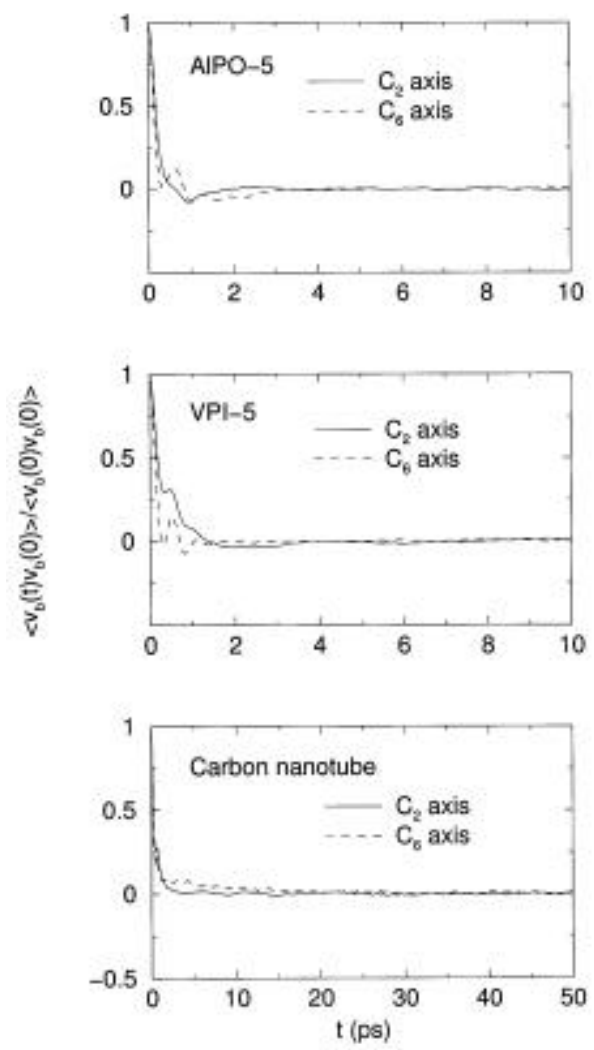

Figure 4. VACF for benzene in body-fixed frame calculated along the $C_{6}$ and $C_{2}$ axes in $\mathrm{AlPO}_{4}-5$, VPI-5 and carbon nanotube.

Table 2. Self-diffusion coefficient for benzene in body-fixed frame.

\begin{tabular}{lccc}
\hline & $D_{\perp}^{t}\left(10^{-9} \mathrm{~m}^{2} / \mathrm{s}\right)$ & $D_{\|}^{t}\left(10^{-9} \mathrm{~m}^{2} / \mathrm{s}\right)$ & $\beta=D_{\perp}^{t} / D_{\|}^{t}$ \\
\hline AlPO $_{4}-5$ & 3.91 & 1.54 & 2.54 \\
VPI-5 & 7.18 & 3.92 & 1.83 \\
Carbon nanotube & 13.9 & 39.6 & .0 .35 \\
\hline
\end{tabular}

period. The rotational motion as characterized by angular velocity autocorrelation function is different around $C_{6}$ axis and $C_{2}$ axis in VPI-5. On the other hand, the difference between the rotational motion around $C_{6}$ and $C_{2}$ axes is less marked in case of carbon nanotube and $\mathrm{AlPO}_{4}-5$. The rotational diffusion coefficient is calculated from,

$$
\begin{aligned}
& D_{\|}^{r}=\int_{0}^{\infty}\left\langle\omega_{\|}(0) \omega_{\|}(t)\right\rangle \mathrm{d} t, \\
& D_{\perp}^{r}=\int_{0}^{\infty}\left\langle\omega_{\perp}(0) \omega_{\perp}(t)\right\rangle \mathrm{d} t,
\end{aligned}
$$



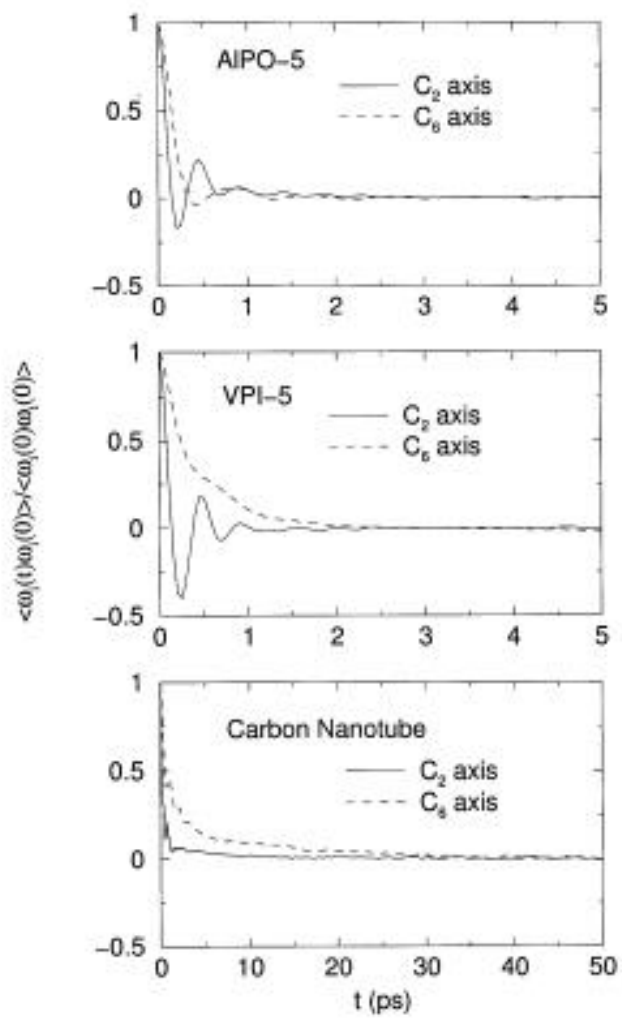

Figure 5. Angular velocity correlation function of benzene for angular velocity components along $C_{6}$ and $C_{2}$ axes in $\mathrm{AlPO}_{4}-5$, VPI-5 and carbon nanotube.

Table 3. Rotational diffusion coefficient for benzene.

\begin{tabular}{llll}
\hline & $D_{\perp}^{r}\left(\mathrm{ps}^{-1}\right)$ & $D_{\|}^{r}\left(\mathrm{ps}^{-1}\right)$ & $\beta=D_{\|}^{r} D_{\perp}^{r}$ \\
\hline AlPO-5 & $0 \cdot 37$ & $0 \cdot 22$ & 0.59 \\
VPI-5 & $0 \cdot 092$ & 0.52 & $5 \cdot 70$ \\
Carbon nanotube $^{\text {Liquid benzene (experimental) }}{ }^{18}$ & $2 \cdot 19$ & $4 \cdot 38$ & $2 \cdot 00$ \\
Liquid benzene (simulation) $^{14}$ & $0 \cdot 07-0.15$ & $0 \cdot 1-0.2$ & $1.42-1 \cdot 33$ \\
\hline
\end{tabular}

where $\omega_{1}$ is the component of angular velocity parallel to $C_{6}$ axis and $\omega_{1}$ is the component along $C_{2}$ axis. The values of rotational diffusivities thus obtained are listed in table 3. Integration is performed up to 25 ps for $\mathrm{AlPO}_{4}-5$ and VPI-5 and $50 \mathrm{ps}$ for carbon nanotube. Also listed are the values obtained by Linse et $a l^{14}$ for liquid benzene at $312 \mathrm{~K}$ as well as the experimentally obtained values.

From table 3 it can be seen that $D_{\|}^{r}$ and $D_{\perp}^{r}$ for benzene in $\mathrm{AlPO}_{4}-5$ are of the same order of magnitude as simulated liquid benzene. In case of carbon nanotube the values of rotational diffusivities are higher by more than an order of magnitude as compared to 
liquid benzene. In VPI-5, $D_{\perp}^{r}$ is an order of magnitude lower as compared to liquid benzene values of rotational diffusivity.

More importantly, the ratio $\eta=D_{\|}^{r} / D_{\perp}^{r}$ which is listed in table 3 shows interesting trends. For liquid benzene, the ratio of 1.93 obtained from simulation is in agreement with the anisotropy in the molecular diameter of benzene. When benzene is confined within a host matrix, the rotational motion is altered. In carbon nanotube that has uniform diameter and smooth walls, the anisotropy as measured by $\eta$ is similar to what is obtained in liquid benzene. In VPI-5 there is an enhancement of anisotropy to 5.7 as compared to the liquid benzene value, 1.93, obtained from MD. This is presumably because, benzene in VPI-5 remains in a parallel orientation. This orientation of benzene makes it more difficult to rotate around $C_{2}$ axis as compared to $C_{6}$ axis. In $\mathrm{AlPO}_{4}-5$, the ratio $\eta$ is 0.59 which implies that rotation around $C_{2}$ axis is easier than rotation around $C_{6}$. Thus, it appears that the presence of the host can act in opposite ways. In VPI-5, the anisotropy is enhanced while in $\mathrm{AlPO}_{4}-5$ the anisotropy is reduced.

A careful look at the channel diameters and the interaction parameter between benzene and host atom (oxygen in case of VPI-5, $\mathrm{AlPO}_{4}-5$ and carbon in case of carbon nanotube is worthwhile. Table 4 lists the dimension of the narrowest and the widest part of the channel existing in the three different host systems. The levitation ratio ${ }^{15}$,

$$
\gamma=2 \cdot 2^{1 / 6} \sigma_{g h} / \sigma_{w}
$$

where $\sigma_{g h}$ is the hydrogen-host interaction parameter and $\sigma_{w}$ is the window diameter defined as the centre to centre distance between diagonal atoms of the channel.

From tables 3 and 4 it is not clear that there is a direct relationship between the anisotropy and the channel dimension. But within aluminophosphates $\left(\mathrm{AlPO}_{4}-5\right.$ and VPI$5)$ it appears that when benzene is confined in a channel of comparable dimension $(\gamma \rightarrow 1)$, the rotational anisotropy is reduced.

Reorientational dynamics could be obtained in terms of the Legendre polynomials $P_{L}(\cos (\phi(t))$ where $\phi(t)$ is the angle between the selected axis at time zero and at a time $t$. Here we report the reorientational correlation function of the $C_{6}$ and the $C_{2}$ axes. Figure 6 shows $\ln \left(\left\langle P_{2}(\cos \phi)\right\rangle\right)$. In $\mathrm{AlPO}_{4}-5$ and carbon nanotube, after the first inertial decay, there is a fast initial decay which is followed by a slow decay. In VPI-5, the initial fast decay is not so pronounced and therefore is closer to what is found in bulk benzene. Assuming an exponential decay, the reorientational correlation times can be obtained from the slope of the long time (fitted to the curve between 20 and $40 \mathrm{ps}$ data) behaviour of the $\ln \left(\left\langle P_{2}(\cos \phi)\right\rangle\right)$. These are listed in table 5 for $C_{6}$ and $C_{2}$ axes rotations. The reorientational

Table 4. Dimensions of the 1D channel and $\gamma$ values for benzene.

\begin{tabular}{llll}
\hline & $\sigma_{w}(\AA)$ & $2^{1 / 6} 2 \sigma_{g h}(\AA)$ & \multicolumn{1}{c}{$\gamma$} \\
\hline AlPO $_{4}-5$ & 10.02 & $11.05(9.52)$ & $1 \cdot 10(0.95)$ \\
& 11.37 & $11.05(9.52)$ & $0.97(0.84)$ \\
VPI-5 & $14.44,16.31$ & $11.05(9.52)$ & $0.77(0.66), 0.68(0.58)$ \\
& $15.92,16 \cdot 31$ & $11.05(9.52)$ & $0.69(0.60), 0.68(0.58)$ \\
Carbon nanotube & $10 \cdot 80$ & $12.03(10.50)$ & $1.02(0.97)$ \\
\hline
\end{tabular}

The quantities in parentheses are calculated from $\sigma_{\mathrm{C}-\mathrm{O}}$ for $\mathrm{AlPO}_{4^{-}} 5$ and VPI-5 and using $\sigma_{\mathrm{C}-\mathrm{C}_{\mathrm{n}}}$ for carbon nanotube. 


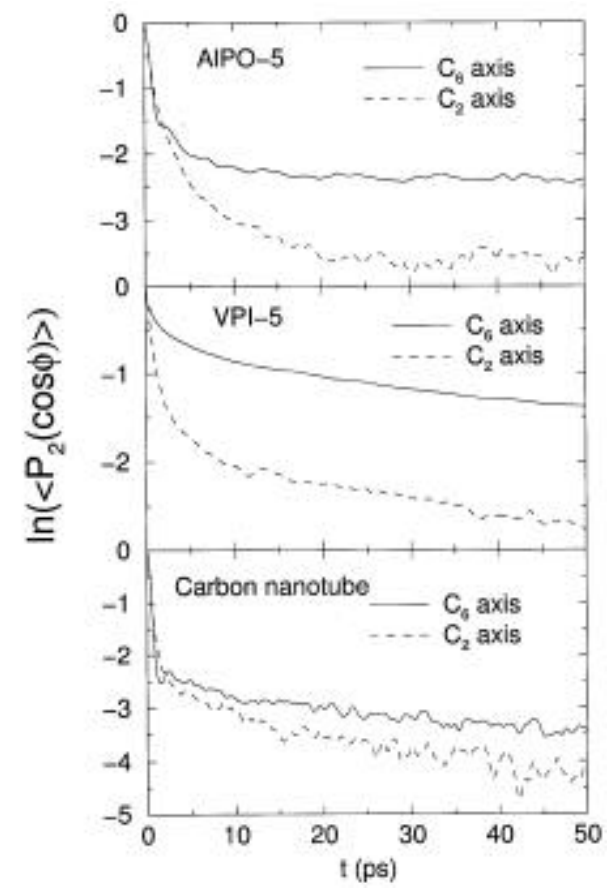

Figure 6. Reorientational correlation function $\left\langle P_{2}(\cos \phi)\right\rangle$ calculated for $C_{6}$ and $C_{2}$ axes of benzene inside $\mathrm{AlPO}_{4}-5$, VPI-5 and carbon nanotube.

Table 5. Reorientational correlation time (ps) of benzene.

\begin{tabular}{lrr}
\hline & $C_{6}$ axis & \multicolumn{1}{c}{$C_{2}$ axis } \\
\hline AlPO $_{4}-5$ & $418 \cdot 1$ & $277 \cdot 3$ \\
VPI-5 & $74 \cdot 9$ & $51 \cdot 1$ \\
Carbon nanotube & $50 \cdot 9$ & $31 \cdot 1$ \\
\hline
\end{tabular}

correlation time of $C_{6}$ axis is always larger than that for $C_{2}$ axis. This is similar to what is observed in bulk benzene where Linse et $^{14}{ }^{14}$ also found that the reorientation around the $C_{6}$ is slower than around $C_{2}$.

The initial fast decay regime is shown up to 3 ps in figure 7 . From figure 7 , it is seen that in the case of VPI-5, the slope for $C_{6}$ is seen to be lower than for $C_{2}$ axis similar to what is found in bulk benzene ${ }^{14}$. However, in $\mathrm{AlPO}_{4}-5$ and carbon nanotube, the decay of reorientational correlation for the $C_{6}$ axis is faster than for $C_{2}$ axis. This is surprising and is against what one expects from a knowledge of the molecular geometry of benzene. It appears that the rather narrow channel affects the reorientational dynamics of benzene strongly enough to even reverse what one expects on the basis of the molecular geometry.

\subsection{Methane and neopentane in zeolite $\mathrm{A}$ and $\mathrm{NaY}$}

Methane is the simplest member of the hydrocarbons as well as the alkane family. Since hydrocarbons obtained from oil fields are processed on large scale by the use of zeolites 


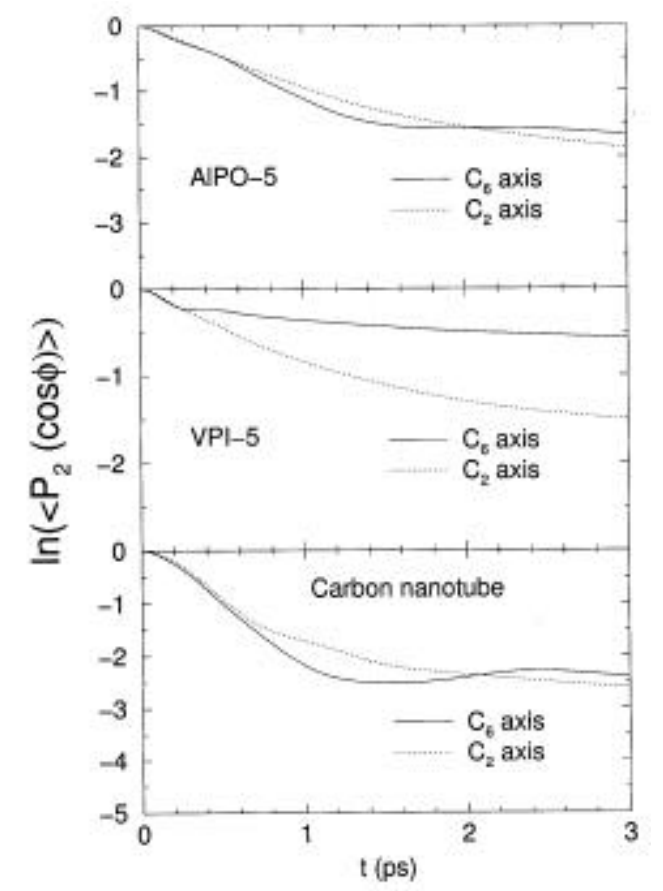

Figure 7. Reorientational correlation function $\left\langle P_{2}(\cos \phi)\right\rangle$ calculated for $C_{6}$ and $C_{2}$ axes of benzene over $1-3$ ps range. This shows the initial fast decay of correlations apart from the inertial decay.

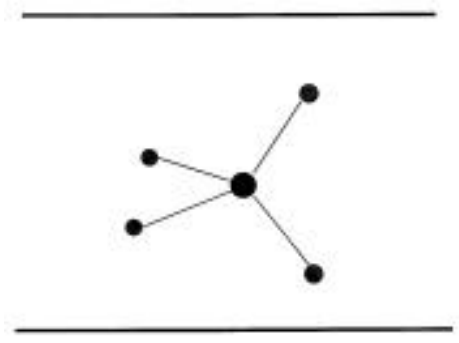

(a)

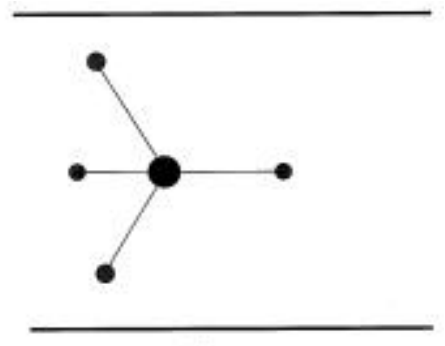

(b)

Figure 8. A schematic representation of orientation of methane with respect to the channel axis in (a) $2+2$ and (b) $1+3$ orientation. In the case of zeolites $\mathrm{Y}$ and $\mathrm{A}$, the vector perpendicular to the window plane is parallel to the channel axis.

in refineries, the study of methane in zeolites is important. Analysis of methane orientation through the 8-ring window which is the bottleneck in the path of diffusion indicated that first, two hydrogens pass through the window, followed by carbon and by the other two hydrogens. This is termed $2+2$ orientation. If methane passes through with a single methane pointing toward the window and if this is followed by carbon and then the three hydrogens (see figure 8 ) then this is termed $1+3$. The former orientation is like that of a pair of scissors while the latter is like an inverted umbrella. Classical MD 


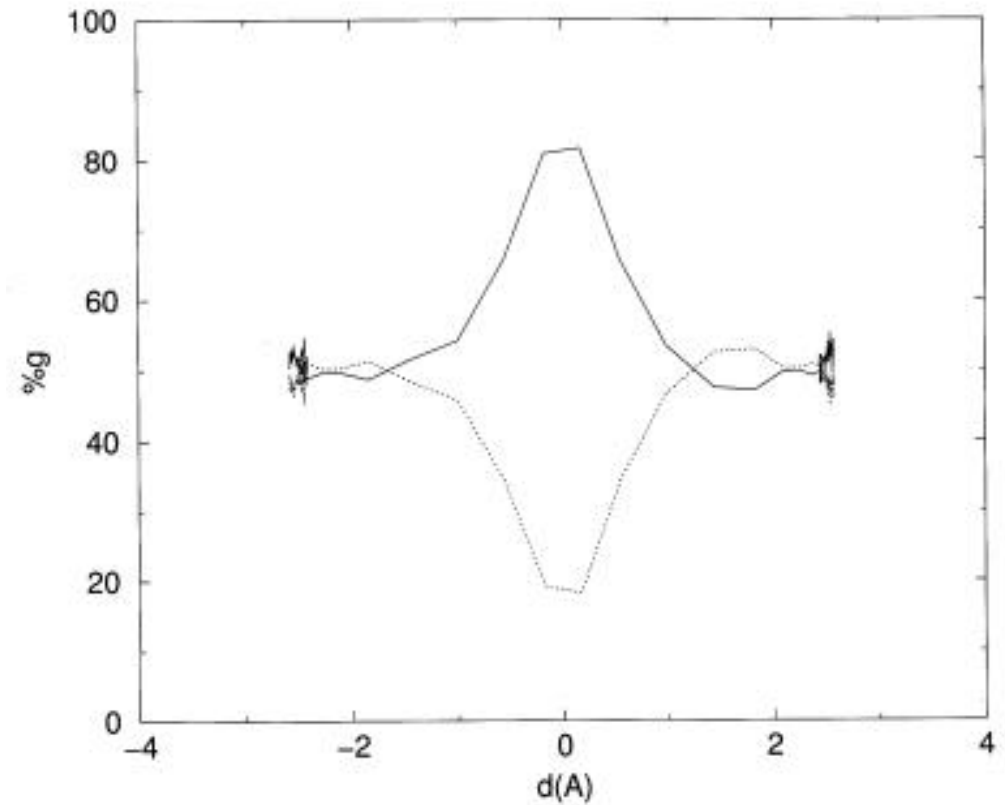

Figure 9. Percentage of methane in $2+2$ orientation while passing through the 8 ring window in zeolite $\mathrm{NaCaA}$. The average over MD trajectories is reported and the abscissa is the distance from the window plane. $d$ is considered to be negative before the methane passes through the window.
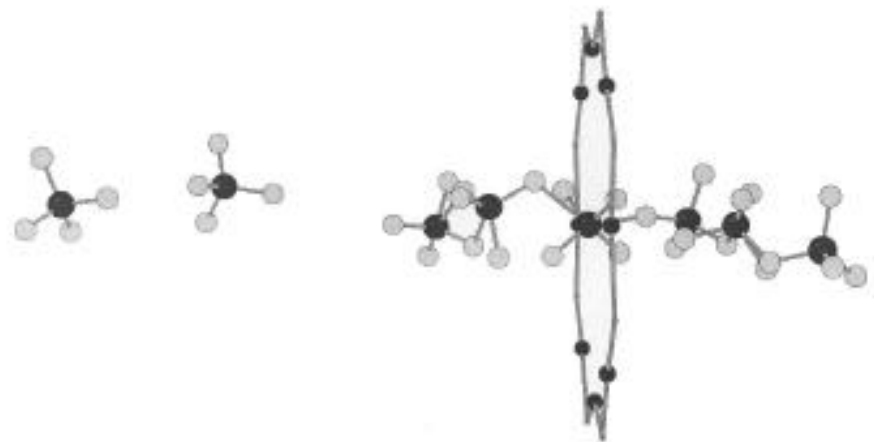

Figure 10. One of MD trajectories of methane passing through the 8-ring window is depicted here as snapshots at various MD time steps.

suggest that $2+2$ is the predominant orientation while passing through the window (see figures 9 and 10). Inside the $\alpha$ cage no such orientational preference is observed and both $1+3$ and $2+2$ are equally populated. The methanes are classified to be in either $1+3$ or $2+2$ based on the value of $\theta_{\min }$. Here, $\theta_{\min }$ is the minimum of the four angles 


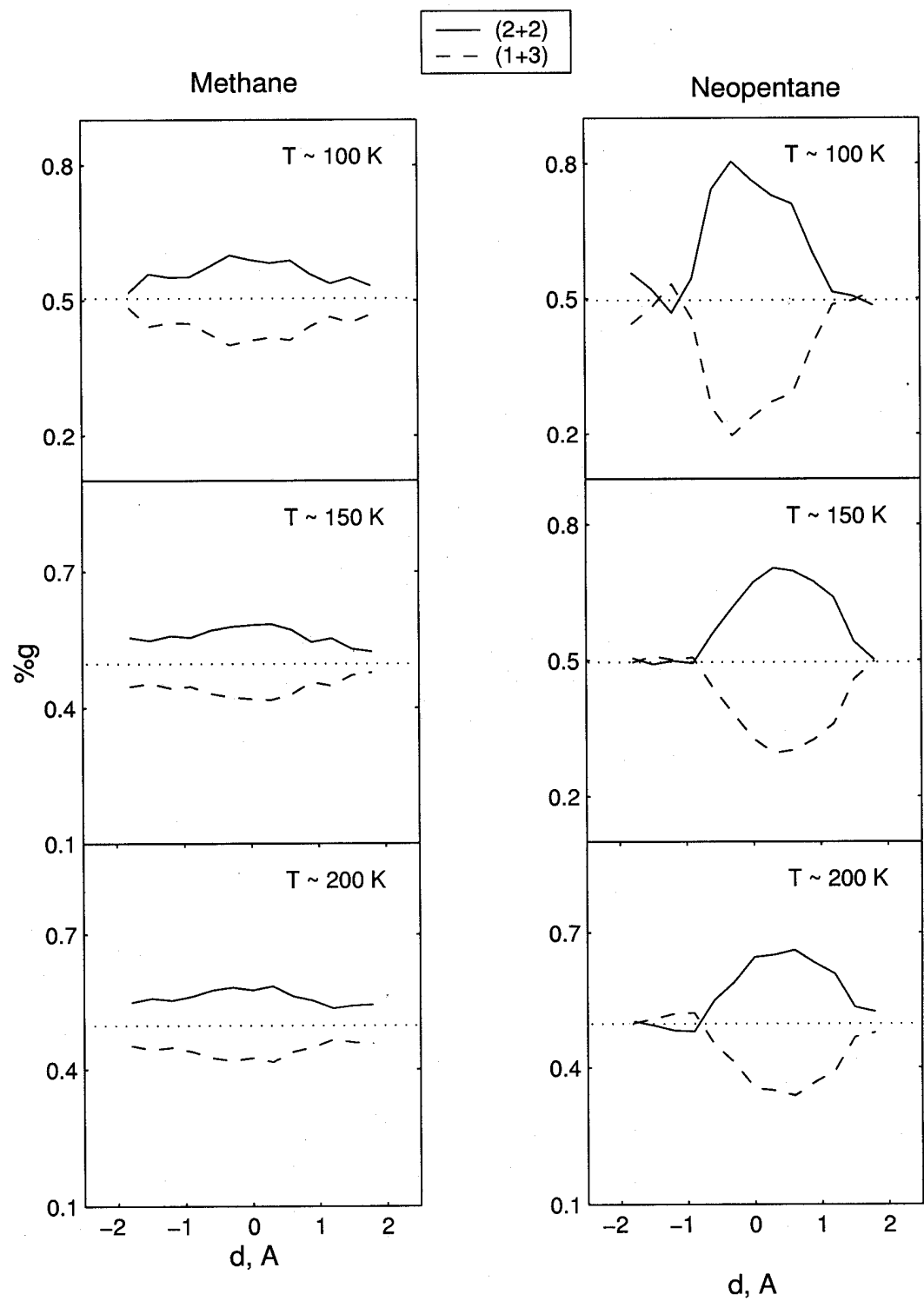

Figure 11. Percentage of methane and neopentane in $2+2$ orientation and $1+3$ orientation during its passage through the bottleneck (12-ring window) in zeolite NaY. Values reported are the MD averages carried out over the whole run.

that the four $\mathrm{C}-\mathrm{H}$ bonds make with the vector perpendicular to the window plane. For $1+3,0<\theta_{\min }<\Theta_{T_{d}} / 4$ and $2+2, \Theta_{T_{d}} / 4<\theta_{\text {min }}<\theta_{T_{d}}$.

In contrast to these findings, methane shows no strong preference for $2+2$ orientation in zeolite $\mathrm{NaY}$ where the bottleneck is the 12-ring window interconnecting two neighbouring cages (see figure 11). Neopentane in $\mathrm{NaY}$, on the other hand shows stronger preference for $2+2$ orientation (see figure 10). This suggests whenever $\gamma$ is 
nearer to unity which corresponds to the condition for levitation $2+2$ is preferred. Thus, not only is the levitation ratio of considerable significance when we discuss the translational motion but it is of equal significance when we speak of rotational motion.

\subsection{Methane in $\mathrm{AlPO}_{4}-5$ and carbon nanotube}

The orientation of methane with respect to the channel axis can be characterized by calculating the angle that each of $\mathrm{C}-\mathrm{H}$ bonds $\left(C_{3}\right.$ axes) subtends to the channel axis. Minimum of these four angles, $\boldsymbol{\theta}_{\min }$, is found out. This $\boldsymbol{\theta}_{\min }$ is in the range 0 to $\boldsymbol{\theta}_{T_{d}} / 2$, where $\theta T_{d}$ is the tetrahedral angle. $\theta_{\min }$ helps in identifying two orientations of methane with respect to channel axis. When $\Theta_{\min }=\Theta_{T_{d}} / 2$ or a $C_{2}$ axis of methane is parallel to channel axis, we term this orientation as perfect $2+2$ orientation and when $\theta_{\min }=0$ or a $C_{3}$ axis parallel to channel axis we term this orientation as perfect $1+3$ orientation. A schematic representation of $1+3$ and $2+2$ orientations of methane with respect to a channel are as shown in figure 8 . Orientations were classified as $1+3$, intermediate and $2+2$ if $0<\theta_{\min }<\theta_{T_{d}} / 6, \theta_{T_{d}} / 6<\theta_{\min }<\theta_{T_{d}} / 3$ and $\theta_{T_{d}} / 3<\theta_{\min }<\theta_{T_{d}} / 2$ respectively.

We have calculated the number of methane molecules in each of the above orientation states as a function of position of centre of mass of methane along the channel, $z$ and radial distance of centre of mass of methane from channel axis, $r$ denoted by $n(z)$ and

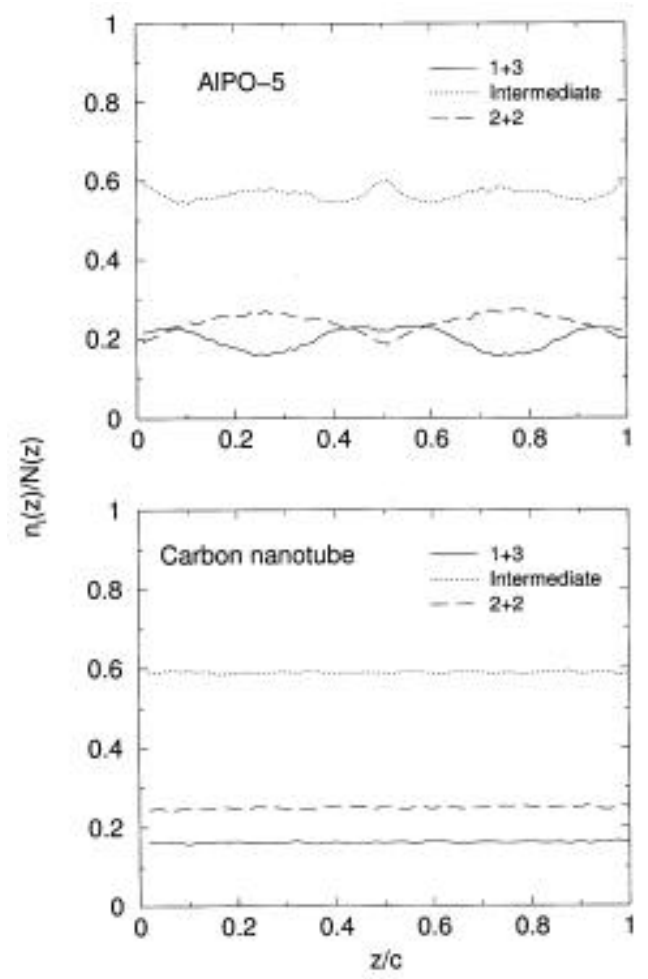

Figure 12. The ratio $n(z) / N(z)$ as a function of position of centre of mass of methane along the channel. $n(z)$ is the number of methane molecules in a given state of orientation at $z$ and $N(z)$ are the total number of molecules at $z . z$ is scaled by the unit cell length $c$ for $\mathrm{AlPO}_{4}-5$ and by $c=49 \cdot 294 \AA$ in case of carbon nanotube. 


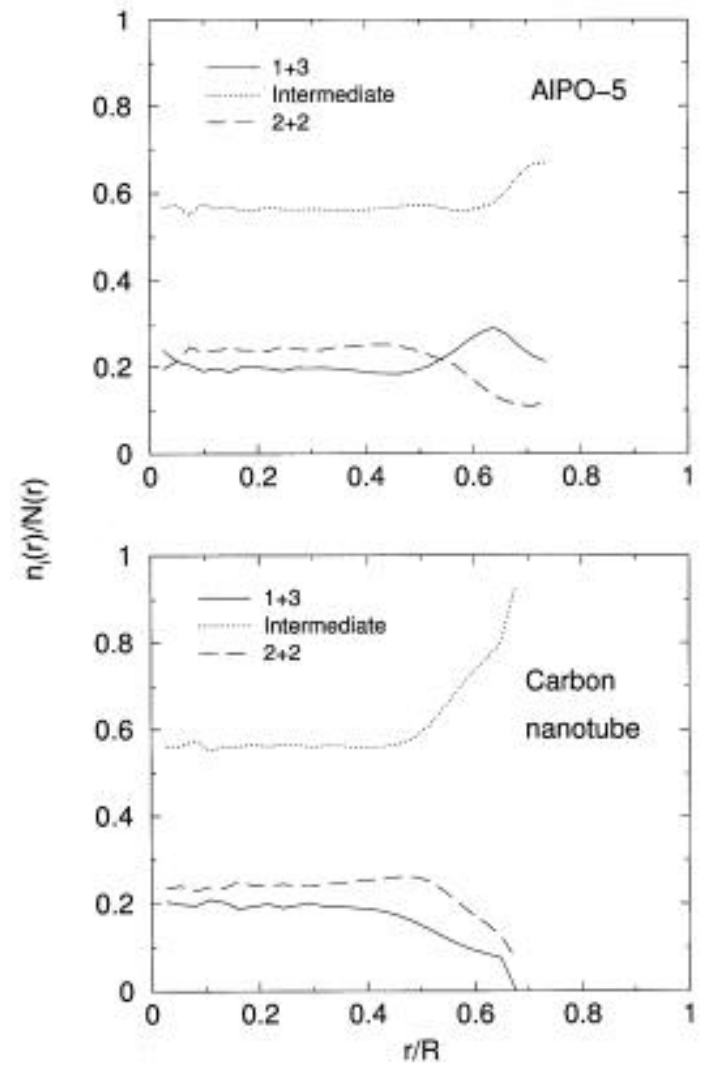

Figure 13. The ratio $n(r) / N(r)$ as a function of radial distance of centre of mass of methane from the channel axis $r . n(r)$ is the number of methane molecules in a given state of orientation at $r$ and $N(r)$ are the total number of molecules at $r . r$ is scaled by radius $R$ of the corresponding channel.

$n(r)$ respectively. In figures 12 and 13 the ratios $n(z) / N(z)$ and $n(r) / N(r)$ are plotted, where $N(z)$ and $N(r)$ are the sums of the total number of molecules in each of the orientation state at given $z$ and $r$ values. Figure 12 shows that the intermediate state is the most populated state at all values of $z$ in both $\mathrm{AlPO}_{4}-5$ and carbon nanotube. In carbon nanotube the $2+2$ state dominates over the $1+3$ state throughout the length of the channel. However, in $\mathrm{AlPO}_{4}-5$ the modulation of diameter of the channel gives rise to contrasting behaviour. At the narrow parts of the channel, $z / c \approx 0 \cdot 25$ and $0.75,2+2$ state is more populated than $1+3$ state, while at the wider parts of the channel it is $1+3$ state that is more populated than $2+2$ state.

Figure 13 shows that intermediate state of orientation of methane is the most populated among all orientation states for all values of $r$ for both the hosts. In carbon nanotube $2+2$ state is more populated as compared to $1+3$ state for all values of $r$. In $\mathrm{AlPO}_{4}-5$ near the centre of the channel $2+2$ state is populated more than $1+3$ state while near the wall of the channel it is $1+3$ state that is populated more than $2+2$ state.

Above results suggest that there exists a correlation between channel diameter and the orientation of methane with respect to the channel axis. Table 6 lists diameter of the channel in $\mathrm{AlPO}_{4}-5$ and carbon nanotube along with the levitation ratio ${ }^{15}$ defined as, 
Table 6. Dimensions of the 1D channel and $\gamma$ values of methane.

\begin{tabular}{lccc}
\hline & $\sigma_{w}(\AA)$ & $2\left(2^{1 / 6} \sigma_{g h}+l\right)(\AA)$ & $\gamma$ \\
\hline $\mathrm{AlPO}_{4}-5$ & $10 \cdot 02$ & 8.196 & 0.817 \\
Carbon nanotube & 11.37 & 8.196 & 0.720 \\
\hline
\end{tabular}

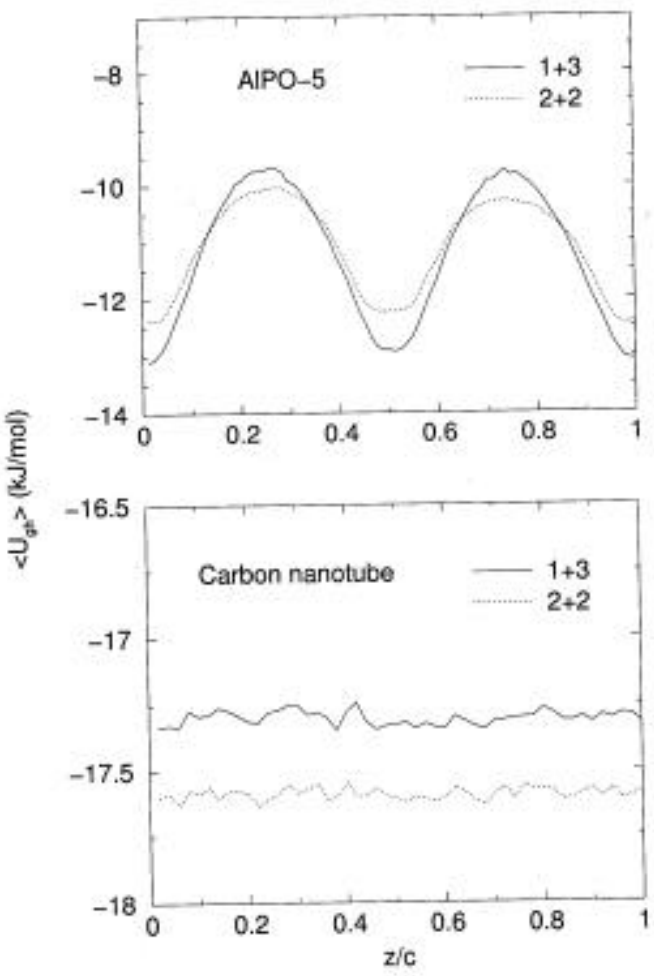

Figure 14. $\left\langle U_{g h}\right\rangle$ as a function of position of centre of mass of methane along the channel $z$ for $1+3$ and $2+2$ orientation. $z$ is scaled by unit cell length for $\mathrm{AlPO}_{4}-5$ and by $c=49 \cdot 294 \AA$ in case of carbon nanotube.

$$
\gamma=\frac{2\left(2^{1 / 6} \sigma_{g h}+l\right)}{\sigma_{w}}
$$

where $\sigma_{g h}$ is hydrogen-host interaction parameter, $\sigma_{w}$ is the window diameter defined as the distance between centres of diagonal atoms of a channel and $l$ is the $\mathrm{C}-\mathrm{H}$ bond length. From table 6 it is clear that for values of $\gamma$ close to 1 (in close fitting parts of $\mathrm{AlPO}_{4}-5$ or in narrow channel like carbon nanotube), more number of methane molecules have $2+2$ orientation than $1+3$ orientation. Similarly, in an earlier study of methane in $\mathrm{NaCaA}^{16}$ it was observed that methane assumes $2+2$ orientation at the 8 -ring window connecting two cages as it passes from one cage to another. 


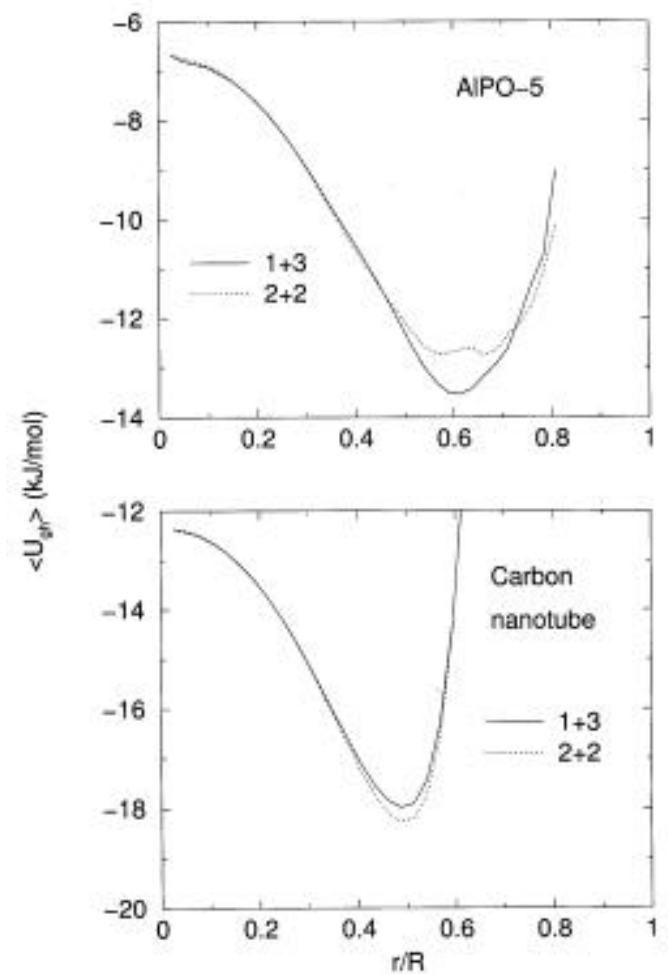

Figure 15. $\left\langle U_{g h}\right\rangle$ as a function of radial distance of centre of mass of methane from the channel axis $r$ for $1+3$ and $2+2$ orientation. $r$ is scaled by radius of respective channel $R$.

The methane-host interaction energy for $2+2$ and $1+3$ orientation as a function of $z$ and $r$ and averaged over the whole MD trajectory, is plotted in figures 14 and 15 respectively. Figure 14 shows that in narrow part of $\mathrm{AlPO}_{4}-5$ channel and in carbon nanotube repulsive contribution to methane-host interaction energy in $1+3$ orientation is more than that in $2+2$ orientation. While in wider part of channel in $\mathrm{AlPO}_{4}-5$ methane can maximize its interaction better in $1+3$ orientation than in $2+2$ orientation. As a function of the radial distance from the axis of the channel, $r$ (see figure 15), methane maximizes its interaction with the host near the wall of the channel in $1+3$ orientation in $\mathrm{AlPO}_{4}-5$ and in $2+2$ orientation in carbon nanotube.

Figure 16 shows the average value of radial distance of centre of mass of methane from the channel axis as a function of its position along the channel. It can be seen that in $\mathrm{AlPO}_{4}-5$ methane is closer to the wall in wider parts of the channel and nearer to the centre of the channel in narrow parts of the channel. In carbon nanotube the average radial distance of both the orientations of methane remains the same along the channel. Among the two orientations, in carbon nanotube, methane in $2+2$ orientation is farther from the channel axis than $1+3$ orientation. In $\mathrm{AlPO}_{4}-5$, in narrow part of the channel the same behaviour as carbon nanotube is seen. The behaviour is reversed when channel diameter increases in wider part of the channel: $2+2$ orientation is closer to the channel axis than $1+3$ orientation. 

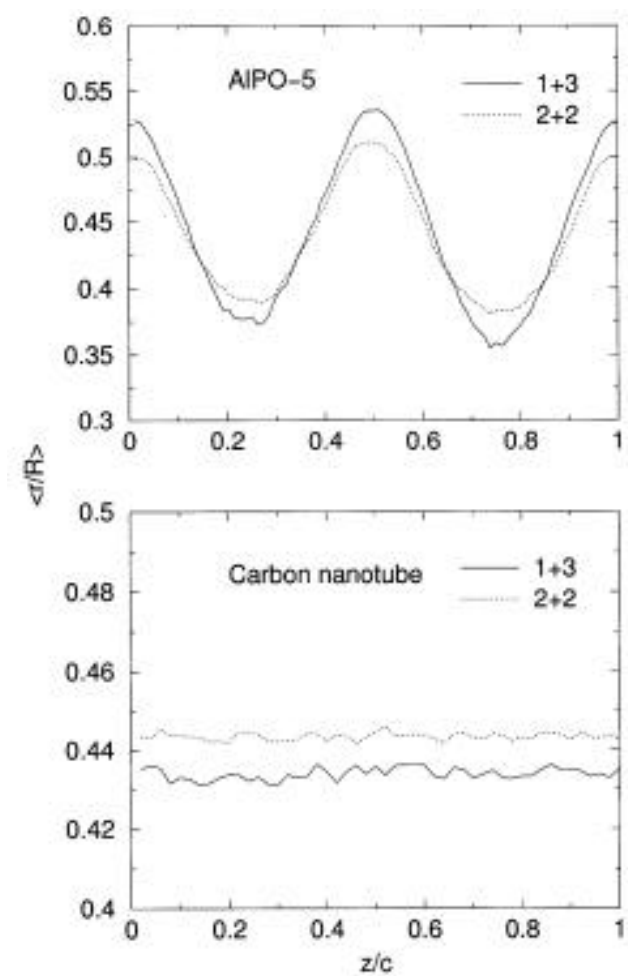

Figure 16. The average radial distance of centre of mass of methane $\langle r\rangle$ from the channel axis as a function of position of centre of mass of methane along the channel $z .\langle r\rangle \mathrm{vs} z$ is plotted for $1+3$ and $2+2$ orientations of methane. $r$ is scaled by the radius of the respective channel and $\mathrm{z}$ is scaled by unit cell length $c$ in $\mathrm{AlPO}_{4}-5$ and by $c=49 \cdot 294 \AA$ in case of carbon nanotube.

These results suggest that the orientation of methane inside a one-dimensional channel is sensitive to the channel diameter. In these simulations the Lennard-Jones parameter $\sigma$ of the host atoms determines the diameter of the channel. Pellenq and Nicholson ${ }^{17}$ recently made an accurate determination of Lennard-Jones parameters between rare gases and silicalite-1 host atoms. Similar studies are desirable for hydrocarbon-zeolite interactions. We hope that results here provide a sensitive and crucial test of these parameters. Experiments which can distinguish between $2+2$ and $1+3$ orientations will be able to yield valuable insight into the accuracy of parameters between $\mathrm{CH}_{4}$ and zeolite.

\section{Conclusions}

In summary, it is shown that under normal conditions motion in confined media exhibit creeping motion rather cartwheel like motion. However, as the study of benzene in $\mathrm{AlPO}_{4}-5$, VPI-5 and carbon nanotube showed, motion within these channel systems can give rise to surprises: under certain conditions, for example, benzene would predominantly diffuse in a direction that is parallel to the $C_{6}$ axis. Note that normally benzene diffuses with its plane in a flat position with respect to the inner surface of the 
cage. In other words, diffusion direction is parallel to $C_{2}$ axis. The diffusion direction is parallel to $C_{6}$ axis when the levitation parameter is close to unity.

Apart from the influence of levitation parameter on translational mobility, the study of benzene in different one-dimensional channels demonstrates that the rotational motion is also strongly a function of the levitation parameter. It is seen that $\eta=D_{\|}^{r} / D_{\perp}^{r}$ is around 1.4 for liquid benzene. In VPI-5 and carbon nanotube $\eta$ is greater than 1 . In contrast, in $\mathrm{AlPO}_{4}-5$, the value is less than 1 showing that diffusion around $C_{6}$ is less facile as compared to $C_{2}$. The reversal of the $D$ values suggests that rotational motion is strongly altered by the host environment.

While the previous study demonstrates that both dynamics of translation and rotation are altered by the host environment, the study of methane and neopentane in A zeolite and $\mathrm{NaY}$ suggests that the orientation of methane show strong preferences. This is found especially when the levitation parameter is near unity. This is the case for methane in $\mathrm{NaCaA}$ while it passes through the 8-ring window, the bottleneck for diffusion but not for methane in $\mathrm{NaY}$ where the bottleneck is the 12-ring window which is much larger in diameter. However, neopentane which is much larger in size exhibits similar trend as methane in A zeolite: preference for $2+2$.

Finally, methane in one-dimensional channels $\mathrm{AlPO}_{4}-5$ and carbon nanotube which have dimensions rather comparable to the size of methane have given interesting insight into the orientational preference of methane and levitation parameter. It is seen that when the channel diameter is rather narrow, preference exists for $2+2$ whereas when the channel diameter is not so narrow the preference is for $1+3$.

\section{Acknowledgements}

Partial financial support from the Council of Scientific and Industrial Research through a research grant as well as through support of a fellowship (to SYB) is gratefully acknowledged. Authors also wish to thank Department of Science \& Technology, New Delhi for financial support.

\section{References}

1. Demontis P and Suffritti G B 1997 Chem. Rev. 972845

2. Bates S P and van Santen R A 1997 Adv. Catal. 421

3. Keil F J, Krishna R and Coppens M O 2000 Rev. Chem. Eng. 1671

4. Pluth J J and Smith J V 1980 J. Am. Chem. Soc. 1024704

5. Fitch A N, Jobic H and Renouprez A 1986 J. Phys. Chem. 901311

6. Richardson Jr. J W, Pluth J J and Smith J V 1986 Acta Crystallogr. C43 1311

7. Crowder C E, Garces J M and Davis M E 1989 Adv. X-ray Crystallogr. 32507

8. Ayappa K G 1998 Langmuir 14880

9. Zhang F 1999 J. Chem. Phys. 1119082

10. Demontis P, Yashonath S and Klein M L 1989 J. Phys. Chem. 935013

11. Saravanan C and Auerbach S M 1999 J. Chem. Phys. 11011000

12. Jousse F., Auerbach S M and Vercauteren D P 2000 J. Phys. Chem. B104 2360

13. Bhide S Y and Yashonath S 2000 J. Phys. Chem. B104 11977

14. Linse P and Engstrom S 1985 Chem. Phys. Lett. 11595

15. Yashonath S and Santikary P 1994 J. Phys. Chem. 986368

16. Chitra R, Anil Kumar A V and Yashonath S 2001 J. Chem. Phys. 11411

17. Pellenq R J-M and Nicholson D 1994 J. Phys. Chem. 9813339

18. McCool M A, Collings A F and Woolf L A 1972 J Chem. Soc., Faraday Trans. I 6895 